\section{AINA NEWS}

\section{Northern Contaminants Program (NCP) Publications Database}

Looking for information about contaminants in the Canadian Arctic? The NCP Publications Database (www. aina.ucalgary.ca/ncp) now contains more than 4100 publications from the program. These records include, but are not limited to, NCP secretariat publications, Arctic Monitory and Assessment Programme (AMAP) reports, and Canadian Arctic Contaminants Assessment Reports (CACAR), books, journal articles, theses, and posters. The NCP's document repository holds many items not otherwise available online. ASTIS is pleased to work with NCP to provide this searchable archive of their publications.

\section{Kluane Lake Research Station News}

The Kluane Lake Research Station (KLRS) opened with COVID-19 safe protocols in place on August 1. The station hosted a small number of researchers from Yukon, Northwest Territories, and British Columbia. Dr. Matthew Ayre, a postdoctoral fellow at AINA, has joined the station management team alongside Dr. Henry Penn and Executive Chef Robert Reich. The team has taken advantage of this period of relative quiet to make numerous improvements to current facilities. Both shower rooms have been refurbished and updates to the kitchen, user office, and storage facilities have been made. Additionally, a new seating and fire pit area has been created near the mess hall (Fig. 1). The station will be transitioning to winter operations beginning in October.

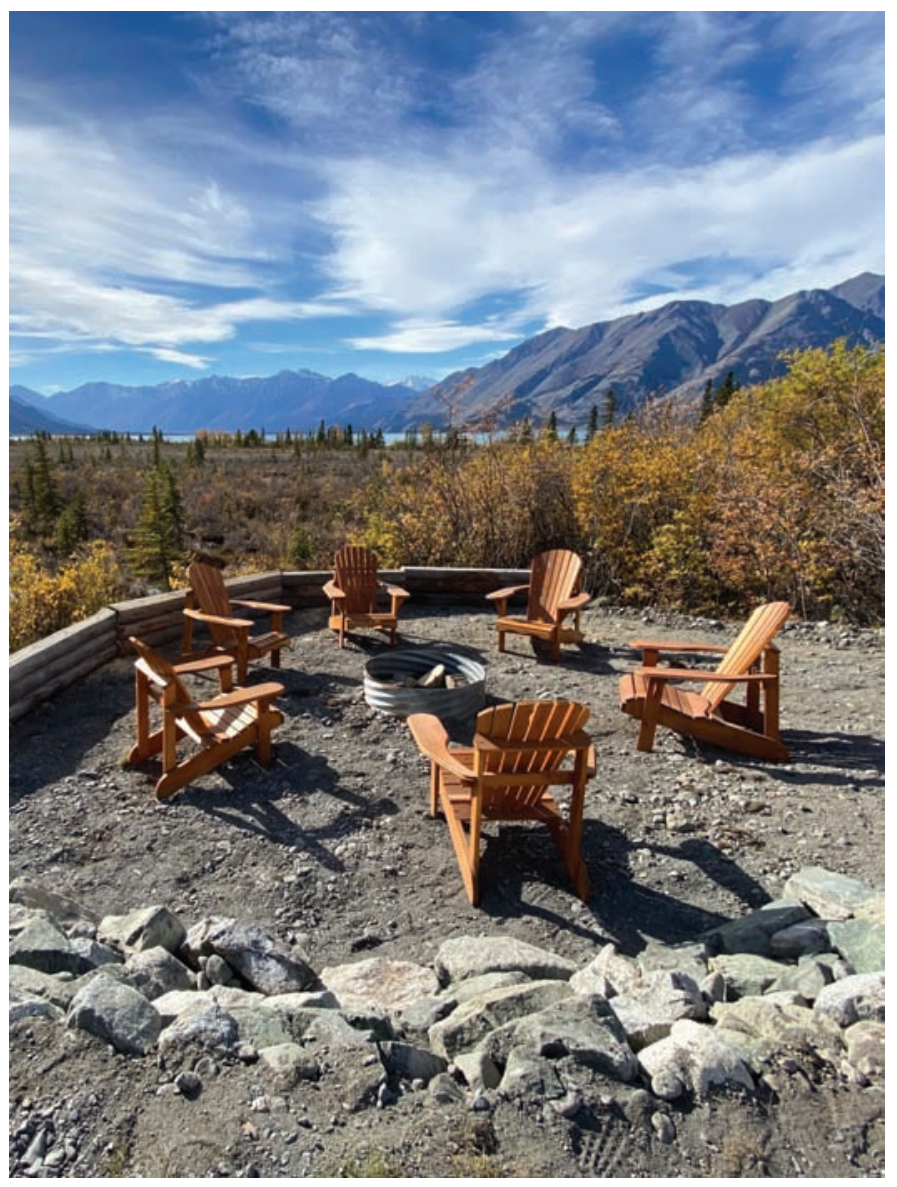

Fig. 1. View from the new seating area at KLRS showing fall colours. (Photo by Matt Ayre). 